\title{
Research Article \\ Exact Solitary Wave Solution in the ZK-BBM Equation
}

\author{
Juan Zhao and Wei Li \\ College of Science, Beijing University of Chemical Technology, Beijing 100029, China \\ Correspondence should be addressed to Juan Zhao; kekezj123@126.com
}

Received 16 June 2014; Revised 4 August 2014; Accepted 18 August 2014; Published 31 August 2014

Academic Editor: Giovanni P. Galdi

Copyright (C) 2014 J. Zhao and W. Li. This is an open access article distributed under the Creative Commons Attribution License, which permits unrestricted use, distribution, and reproduction in any medium, provided the original work is properly cited.

\begin{abstract}
The traveling wave solution for the ZK-BBM equation is considered, which is governed by a nonlinear ODE system. The bifurcation structure of fixed points and bifurcation phase portraits with respect to the wave speed $c$ are analyzed by using the dynamical system theory. Furthermore, the exact solutions of the homoclinic orbits for the nonlinear ODE system are obtained which corresponds to the solitary wave solution curve of the ZK-BBM equation.
\end{abstract}

\section{Introduction}

Nonlinear dispersive equations are important models to describe a lot of physical phenomena and engineering problems. Among all the nonlinear phenomena exhibited by the systems, the solitary wave is one of the most interesting motions, which is a special wave related to many physical and mathematical problems such as turbulence and chaos. But it is usually not a simple work to find the solitary wave in a nonlinear dispersive equation. Several methods have been introduced to find a solitary wave in those equations, such as the tanh-sech method, the sine-cosine algorithm, the homogeneous balance method, and the inverse scattering method. See [1-3] for details.

Among all the nonlinear dispersive systems, the $\mathrm{KdV}$ equation and the dissipative Burgers equation have been paid more attention by many authors and their general wave solution and the solitary wave solution have been well discussed. See $[4,5]$ for details. Some general forms of the KdV equation have also been introduced. In 1972, the Benjamin-Bona-Mahony equation has been proposed as a model for propagation of long waves, where the nonlinear dispersion is incorporated. See [6] for details. Its assumption is similar to $\mathrm{KdV}$ equation and the model is as follows:

$$
u_{t}+u_{x}-a\left(u^{2}\right)_{x}-b u_{x x t}=0 .
$$

In 1974, Zakharov and Kuznetsov proposed an equation to govern the behavior of weakly nonlinear ion-acoustic waves in plasma comprising cold ions and hot isothermal electrons. See [7] for details. The ZK equation is

$$
u_{t}+u u_{x}+u_{x x x}+u_{x y y}=0 .
$$

In 2005, Wazwaz structured an equation by combining the $\mathrm{BBM}$ equation with the $\mathrm{ZK}$ equations, that is, the ZK-BBM equation:

$$
u_{t}+u_{x}-a\left(u^{2}\right)_{x}-\left(b u_{x t}+k u_{y t}\right)_{x}=0 .
$$

The dynamics of the ZK-BBM equation has been discussed and the existence of the solitary wave has been considered in that paper. But the exact solutions of the solitary wave are still unknown, which would be more important to find out other dynamics of the ZK-BBM equation.

In the paper, we will attempt to find the solitary wave in the ZK-BBM equation. The traveling wave for this equation, $u=\varphi(m x+n y-c t)$, is considered in this study, which is governed by a nonlinear ODE system, whose homoclinic orbit is just the solitary wave of the ZK-BBM equation. The bifurcation structure of this dynamical system is discussed for the wave speed $c$. Then, the dynamics of the ZK-BBM equation is discussed and the explicit expressions of the solitary wave solutions are obtained.

This paper is arranged as follows. In Section 2, the governed equation for the traveling wave for the ZK-BBM equation is introduced and the Hamiltonian structure of the system is considered. In Section 3, the solitary wave for the ZK-BBM equation with $g=0$ is considered. In Section 4, 
the solitary wave for the ZK-BBM equation with $g \neq 0$ is discussed and the conclusion of this study is summarized in Section 5.

\section{Traveling Wave Solution}

Assume that the nonlinear ZK-BBM equation

$$
u_{t}+u_{x}-a\left(u^{2}\right)_{x}-\left(b u_{x t}+k u_{y t}\right)_{x}=0
$$

admits a traveling wave solution in form $u=\varphi(\xi), \xi=$ $m x+n y-c t$, where the wave speed $c$ is constant. Equation (4) becomes

$$
c m(b m+k n) \varphi^{\prime \prime \prime}-2 a m \varphi \varphi^{\prime}+(m-c) \varphi^{\prime}=0 .
$$

Integrating (5), we get

$$
c m(b m+k n) \varphi^{\prime \prime}-a m \varphi^{2}+(m-c) \varphi=g,
$$

where $g$ is the integration constant. Letting $v=\varphi^{\prime}$, we get the following planar system:

$$
\begin{aligned}
\varphi^{\prime}= & v, \\
v^{\prime}= & \frac{a}{c(b m+k n)} \varphi^{2}-\frac{(m-c)}{c m(b m+k n)} \varphi \\
& +\frac{g}{c m(b m+k n)} .
\end{aligned}
$$

Obviously, (7) is a Hamiltonian system with energy function:

$$
\begin{aligned}
H(\varphi, v)= & \frac{v^{2}}{2}-\frac{a \varphi^{3}}{3 c(b m+k n)} \\
& +\frac{(m-c) \varphi^{2}}{2 c m(b m+k n)}-\frac{g \varphi}{c m(b m+k n)}=h,
\end{aligned}
$$

where $h$ is the integration constant.

\section{The Solitary Wave with $g=0$}

When the constant $g=0$, system (7) reduces to the following system:

$$
\begin{aligned}
\varphi^{\prime} & =v, \\
v^{\prime} & =\frac{a}{c(b m+k n)} \varphi^{2}-\frac{m-c}{c m(b m+k n)} \varphi .
\end{aligned}
$$

This equation has two fixed points at $O(0,0)$ and $P((m-c) / a m, 0)$. Denote $\varphi_{1}=0, \varphi_{2}=(m-c) / a m$. By the linearized stability theory, we obtain the following conclusions.

Lemma 1. For bm $+k n>0$, system (9) has two fixed points, whose stability with respect to the wave speed $c$ is given by the following.

(i) When $\mathrm{cm}(m-c)<0, O$ is the saddle point and $P$ is a center point.

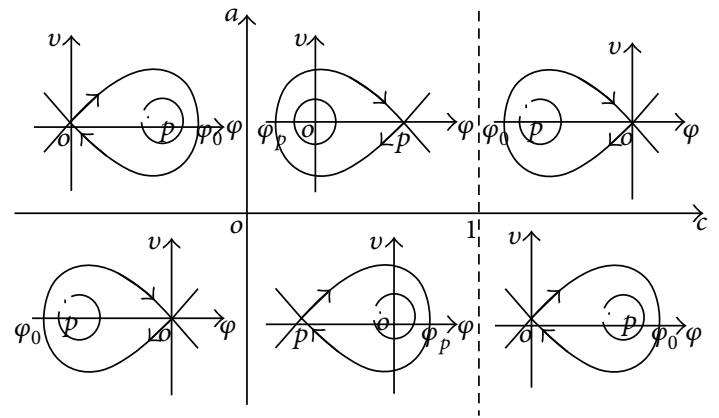

FIgURE 1: The bifurcation phase portraits of system (9) when $b+k>$ 0 and $m=n=1$.

(ii) When $c m(m-c)=0, O$ and $P$ are a degenerate saddle point.

(iii) When $\mathrm{cm}(m-c)>0, O$ is a center point and $P$ is a saddle point.

The bifurcation phase portraits of system (9) are shown in Figure 1.

3.1. The Homoclinic Orbit of Saddle Point O When $\mathrm{cm}(m-c)<$ 0 . By the above analysis, system (9) exhibits a homoclinic orbit $\Gamma_{O}$ connecting to saddle point $O$. By (8), we obtain

$$
v= \pm \sqrt{\frac{2 a}{3 c(b m+k n)} \varphi^{3}-\frac{m-c}{c m(b m+k n)} \varphi^{2}},
$$

where $\varphi \in\left(0, \varphi_{0}\right), \varphi_{0}=3(m-c) / 2 a m$, and $\left(\varphi_{0}, 0\right)$ is another crossover point of $\Gamma_{O}$ and axis $\varphi$.

Using the $\operatorname{ODE} \varphi^{\prime}=v$ with the initial condition $\left.\varphi(\xi)\right|_{\xi=0}=\varphi_{0}$, we obtain the integral form of the homoclinic orbit as follows:

$$
\begin{aligned}
& \int_{\varphi}^{\varphi_{0}} \frac{d \psi}{\sqrt{(2 a / 3 c(b m+k n)) \psi^{3}-((m-c) / c m(b m+k n)) \psi^{2}}} \\
& \quad=\int_{\xi}^{0} d s, \quad \xi<0,
\end{aligned}
$$

$$
\begin{aligned}
& \int_{\varphi}^{\varphi_{0}} \frac{d \psi}{\sqrt{(2 a / 3 c(b m+k n)) \psi^{3}-((m-c) / c m(b m+k n)) \psi^{2}}} \\
& \quad=-\int_{\xi}^{0} d s, \quad \xi>0 .
\end{aligned}
$$


Owing to $(m-c) / c m(b m+k n)<0$, we get integral curve of the homoclinic orbit as

$$
\begin{aligned}
& \left(\frac{c-m}{c m(b m+k n)}\right)^{-1 / 2} \\
& \cdot \ln \mid\left(\sqrt{\frac{2 a \varphi}{3 c(b m+k n)}-\frac{m-c}{c m(b m+k n)}}\right. \\
& \left.\quad-\sqrt{\frac{c-m}{c m(b m+k n)}}\right) \\
& \quad\left(\sqrt{\frac{2 a \varphi}{3 c(b m+k n)}-\frac{m-c}{c m(b m+k n)}}\right. \\
& \left.\quad+\sqrt{\frac{c-m}{c m(b m+k n)}}\right)^{-1} \mid
\end{aligned}
$$

It corresponds to the solitary wave of ZK-BBM equation. Thus, we obtain Theorem 2.

Theorem 2. For the wave speed $c m(c-m)>0$, the solitary wave curve of (4) is

$$
u=\frac{3(m-c)}{a m[\cosh [\mu(m x+n y-c t)]+1]},
$$

where $\mu=\sqrt{(c-m) / c m(b m+k n)}$, and wave crest is $|3(m-c) / 2 a m|$.

\subsection{The Homoclinic Orbit of Saddle Point $P$ When $\mathrm{cm}(m-c)>$} 0 . By the bifurcation analysis, system (9) exhibits a homoclinic orbit $\Gamma_{p}$ connecting to saddle point $p$ in this case. By (8), we obtain

$$
v= \pm \sqrt{\frac{2 a}{3 c(b m+k n)} \varphi^{3}-\frac{m-c}{c m(b m+k n)} \varphi^{2}+2 h_{p}}
$$

where $h_{p}=H\left(\varphi_{2}, 0\right), \varphi \in\left(\varphi_{2}, \varphi_{p}\right), \varphi_{p}=-(m-c) / 2 a m$, and $\left(\varphi_{p}, 0\right)$ is another crossover point of $\Gamma_{p}$ and axis $\varphi$.

Similarly, we obtain the integral form of the homoclinic orbit as follows:

$$
\begin{gathered}
\int_{\varphi}^{\varphi_{p}}\left(\frac{2 a}{3 c(b m+k n)} \psi^{3}-\frac{m-c}{c m(b m+k n)} \psi^{2}\right. \\
\left.+\frac{(m-c)^{3}}{3 a^{2} c m(b m+k n)}\right)^{-1 / 2} d \psi
\end{gathered}
$$

$$
\begin{aligned}
& =\int_{\xi}^{0} d s, \quad \xi<0 \\
& \int_{\varphi}^{\varphi_{p}}\left(\frac{2 a}{3 c(b m+k n)} \psi^{3}-\frac{m-c}{c m(b m+k n)} \psi^{2}\right. \\
& \left.\quad+\frac{(m-c)^{3}}{3 a^{2} c m(b m+k n)}\right)^{-1 / 2} d \psi \\
& =-\int_{\xi}^{0} d s, \quad \xi>0 .
\end{aligned}
$$

In order to obtain solution of (15), we need to find a transformation to transform

$$
\int \frac{d \varphi}{\sqrt{\alpha \varphi^{3}+\beta \varphi^{2}+\gamma \varphi+\theta}}
$$

to

$$
-\int \frac{A}{\sqrt{B^{2}-K}} d B
$$

by rational linear transformation $B=\left(A_{1} \varphi+B_{1}\right) /$ $\left(A_{2} \varphi+B_{2}\right)$, where $K \in R^{+}$.

Substituting rational linear transformation $B$ into (17) and comparing its coefficient with that in (16), we get five relationships:

$$
\left\{\begin{array}{c}
\varphi^{3}: \frac{\varphi^{4}: A_{1}^{2}=K A_{2}^{2}}{A^{2}\left(A_{2} B_{1}-A_{1} B_{2}\right)}=\frac{\alpha}{2} \\
\varphi^{2}: \frac{5 A_{1} B_{2}+A_{2} B_{1}}{A^{2}\left(A_{2} B_{1}-A_{1} B_{2}\right)}=\beta \\
\varphi: \frac{2 B_{2}\left(A_{2} B_{1}+2 A_{1} B_{2}\right)}{A^{2} A_{2}\left(A_{2} B_{1}-A_{1} B_{2}\right)}=\gamma \\
\text { constant }: \frac{B_{2}^{2}\left(A_{2} B_{1}+A_{1} B_{2}\right)}{A^{2} A_{2}^{2}\left(A_{2} B_{1}-A_{1} B_{2}\right)}=\theta \\
\Longrightarrow\left\{\begin{array}{c}
A^{2}=\frac{2 A_{1} A_{2}}{\alpha\left(A_{2} B_{1}-A_{1} B_{2}\right)} \\
\frac{5 A_{1} B_{2}+A_{2} B_{1}}{A_{1} A_{2}}=\frac{2 \beta}{\alpha} \triangleq D_{1} \\
\frac{2 B_{2}\left(A_{2} B_{1}+2 A_{1} B_{2}\right)}{A_{1} A_{2}^{2}}=\frac{2 \gamma}{\alpha} \triangleq D_{2} \\
\frac{B_{2}^{2}\left(A_{2} B_{1}+A_{1} B_{2}\right)}{A_{1} A_{2}^{3}}=\frac{2 \theta}{\alpha} \triangleq D_{3} .
\end{array}\right.
\end{array}\right.
$$

Lemma 3. The conversion conditions of transforming (16) to (17) are

$$
-D_{1}^{3}+9 D_{1} D_{2}-\left(\sqrt{D_{1}^{2}-6 D_{2}}\right)^{3}=54 D_{3} .
$$

The rational linear transformation $B=\left(A_{1} \varphi+B_{1}\right) /$ $\left(A_{2} \varphi+B_{2}\right)$ is then obtained, where $D_{1}=2 \beta / \alpha, D_{2}=2 \gamma / \alpha$, 

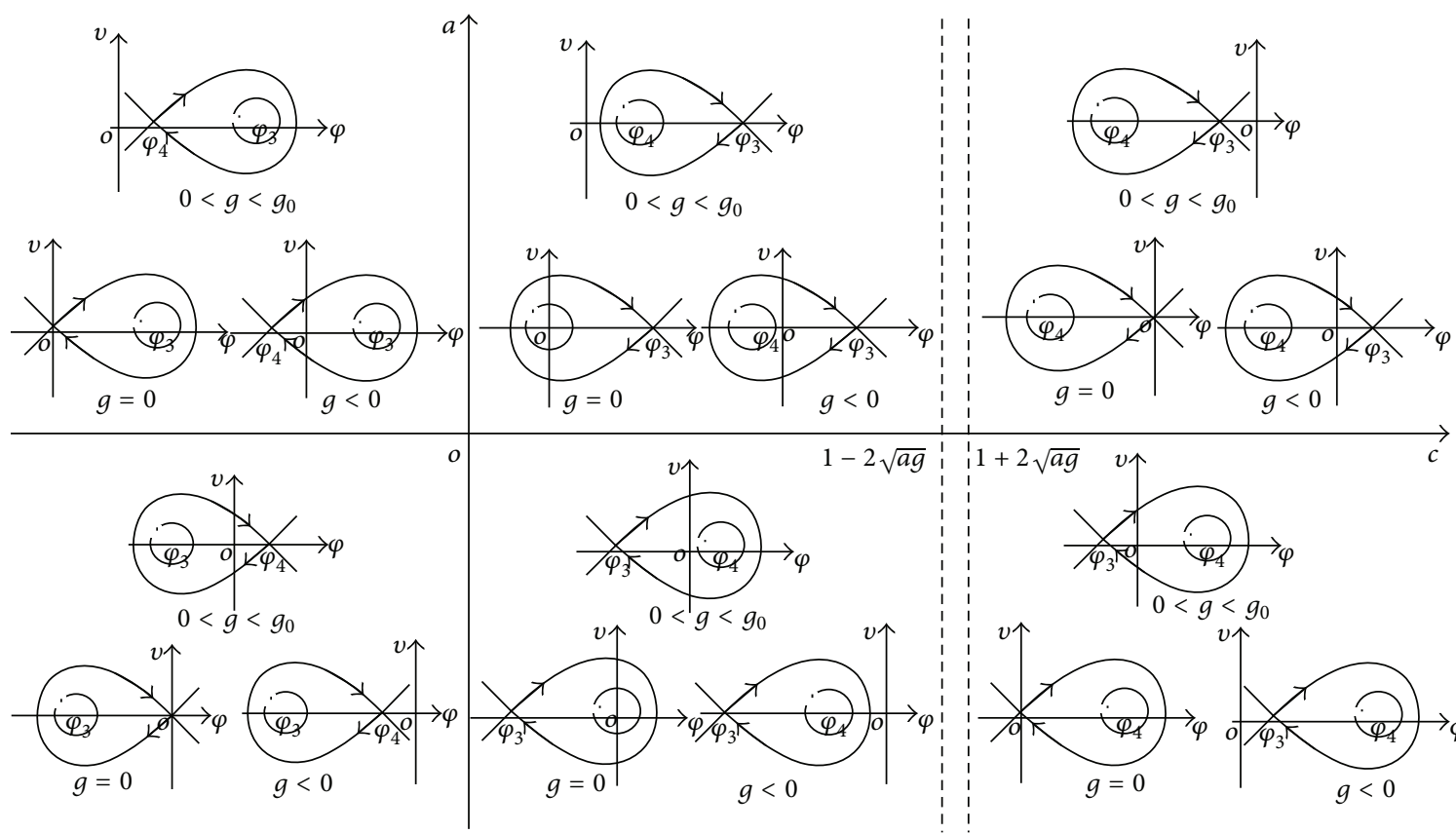

FIgURE 2: The bifurcation phase portraits of system (7) when $b+k>0$ and $m=n=1$.

and $D_{3}=2 \theta / \alpha$. The parameters of transformation $B$ are $A_{1}=$ $\sqrt{K} A_{2}, B_{2}=\left(\left(D_{1}+\sqrt{D_{1}^{2}-6 D_{2}}\right) / 6\right) A_{2}$, and $B_{1}=\sqrt{K} D_{1} A_{2}-$ $5 \sqrt{K} B_{2}$, where $K$ and $A_{2}$ can be chosen freely.

It is easy to examine that the coefficients of $\varphi$ in (15) satisfy the conversion condition (19). Furthermore, set $A_{2}=a$ and $K=4$. The transformation is obtained as $B=(2 a m \varphi+4(m-c)) /(\operatorname{am} \varphi-(m-c))$. Equation $(15)$ becomes

$$
\begin{gathered}
\int\left(\frac{2 a}{3 c(b m+k n)} \varphi^{3}-\frac{m-c}{c m(b m+k n)} \varphi^{2}\right. \\
\left.+\frac{(m-c)^{3}}{3 a^{2} c m(b m+k n)}\right)^{-1 / 2} d \varphi \\
=\int \frac{\sqrt{c m(b m+k n) /(m-c)} d B}{-\sqrt{B^{2}-4}} \\
=\sqrt{\frac{c m(b m+k n)}{m-c} \ln \left|\frac{-B+\sqrt{B^{2}-4}}{2}\right|} .
\end{gathered}
$$

It corresponds to the solitary wave curve of ZK-BBM equation. So we have Theorem 4.

Theorem 4. For the wave speed $c m(c-m)<0$, the solitary wave curve of (4) is

$$
u=\frac{(m-c) \cosh [\kappa(m x+n y-c t)]-2}{a m[\cosh [\kappa(m x+n y-c t)]+1]},
$$

where $\kappa=\sqrt{(m-c) / c m(b m+k n)}$, and the wave crest is $|(m-c) / 2 a m|$.

\section{The Solitary Wave with $g \neq 0$}

The bifurcation of phase portraits of system (7) in this case is considered firstly. Denote $\Delta=(m-c)^{2}-4 a g m$. System (7) does not have fixed point when $\Delta<0$; it has only one fixed point $((m-c) / 2 a m, 0)$ when $\Delta=0$; it has two fixed points $A\left(\varphi_{3}, 0\right)$ and $B\left(\varphi_{4}, 0\right)$ when $\Delta>0$, where $\varphi_{3,4}=$ $((m-c \pm \omega) / 2 a m)(\omega=\sqrt{\Delta} \geq 0)$. By the linearized stability theory, we obtain the following conclusions.

Lemma 5. For $m(b m+k n)>0$, system (7) has two fixed points, whose stability with respect to the wave speed $c$ is given by the following.

(i) When $\mathrm{c} \omega>0, A$ is the saddle point and $B$ is a center point.

(ii) When $\omega=0$ and $c \neq 0, A$ and $B$ are a degenerate saddle point.

(iii) When $c \omega<0, A$ is a center point and $B$ is a saddle point.

The bifurcation phase portraits of system (7) are shown in Figure 2 (where we denote $\left.g_{0}=\left|-(m-c)^{2} / 4 a m\right|\right)$.

By the bifurcation analysis, system (7) exhibits a homoclinic orbit $\Gamma_{A}$ connecting to saddle point $A$. By (8), we obtain

$$
\begin{gathered}
v= \pm\left(\frac{2 a}{3 c(b m+k n)} \varphi^{3}-\frac{m-c}{c m(b m+k n)} \varphi^{2}\right. \\
\left.+\frac{2 g}{c m(b m+k n)} \varphi+2 h_{A}\right)^{1 / 2},
\end{gathered}
$$

where $\varphi \in\left(\varphi_{3}, \varphi_{5}\right), \varphi_{5}=(m-c+2 \omega) / 2 a m, h_{A}=$ $H\left(\varphi_{3}, 0\right)=(m-c+2 \omega)(m-c-\omega) /\left(-24 a^{2} c m(b m+k n)\right)$, 
and $\left(\varphi_{5}, 0\right)$ is another crossover point of $\Gamma_{A}$ and axis $\varphi$. Using the $\operatorname{ODE} \varphi^{\prime}=v$ with the initial condition $\left.\varphi(\xi)\right|_{\xi=0}=\varphi_{5}$, we obtain the integral form of the homoclinic orbit as follows:

$$
\begin{gathered}
\int_{\varphi}^{\varphi_{5}}\left(\frac{2 a}{3 c(b m+k n)} \psi^{3}-\frac{m-c}{c m(b m+k n)} \psi^{2}\right. \\
\left.\quad+\frac{2 g}{c m(b m+k n)} \psi+2 h_{A}\right)^{-1 / 2} d \psi \\
=\int_{\xi}^{0} d s, \quad \xi<0, \\
\int_{\varphi}^{\varphi_{5}}\left(\frac{2 a}{3 c(b m+k n)} \psi^{3}-\frac{m-c}{c m(b m+k n)} \psi^{2}\right. \\
\left.\quad+\frac{2 g}{c m(b m+k n)} \psi+2 h_{A}\right)^{-1 / 2} d \psi \\
=-\int_{\xi}^{0} d s, \quad \xi>0 .
\end{gathered}
$$

It is easy to examine that the coefficients of $\varphi$ in (23) satisfy the conversion conditions (19). Set $A_{2}=a$ and $K=4$. The transformation is obtained as $B=$ $(4 a m \varphi-2(m-c+5 \omega)) /(2 a m \varphi-(m-c-\omega))$. Equation (23) becomes

$$
\begin{aligned}
& \int\left(\frac{2 a}{3 c(b m+k n)} \varphi^{3}-\frac{m-c}{c m(b m+k n)} \varphi^{2}\right. \\
& \left.\quad+\frac{2 g}{c m(b m+k n)} \varphi+\frac{(m-c+2 \omega)(m-c-\omega)}{-12 a^{2} c m(b m+k n)}\right)^{-1 / 2} d \varphi \\
& =\int \frac{\sqrt{c m(b m+k n) / \omega}}{-\sqrt{B^{2}-4}} d B \\
& =\sqrt{\frac{c m(b m+k n)}{\omega}} \ln \left|\frac{-B+\sqrt{B^{2}-4}}{2}\right| .
\end{aligned}
$$

It corresponds to the solitary wave curve of ZK-BBM equation. So we have Theorem 6.

Theorem 6. For the wave speed $0<c<m-2 \sqrt{a g m}$ or $c>$ $m+2 \sqrt{a g m}>0$, the solitary wave curve of (4) is

$$
\begin{aligned}
u=[m-c+5 \omega+(m-c-\omega) \\
\\
\left.\quad \cdot \cosh \left(\sqrt{\frac{\omega}{c m(b m+k n)}}(m x+n y-c t)\right)\right]^{2} \\
\\
\cdot\left(4 a m \operatorname{sech}^{-2}\left(\frac{1}{2} \sqrt{\frac{\omega}{\mathrm{cm}(\mathrm{bm}+\mathrm{kn})}}(\mathrm{mx}+\mathrm{ny}-\mathrm{ct})\right)\right)^{-1},
\end{aligned}
$$

\section{Conclusion}

In this paper, the traveling wave for $\mathrm{ZK}$-BBM equation is considered. The bifurcation phase portraits of nonlinear system governing the traveling wave were studied with respect to the wave speed $c$. All kinds of homoclinic orbits are obtained explicitly for some parameter conditions. Thus, we obtained the exact solitary wave solutions of ZK-BBM equation. In the same time, we give the wave crest value in the different case of wave speed $c$.

It is valuable to point out that the method used in this paper can be widely applied to other nonlinear equations with the similar types. We provide the idea to handle complex integral in order to get the exact solution of homoclinic orbits. Therefore, it lays the foundation for studying the chaotic conditions of ZK-BBM equation in the future.

\section{Conflict of Interests}

The authors declare that there is no conflict of interests regarding the publication of this paper.

\section{References}

[1] A. Wazwaz, "The extended tanh method for new compact and noncompact solutions for the KP-BBM and the ZK-BBM equations," Chaos, Solitons and Fractals, vol. 38, no. 5, pp. 15051516, 2008.

[2] A. Wazwaz, "Solitary wave solutions for modified forms of Degasperis-Procesi and Camassa-Holm equations," Physics Letters A, vol. 352, no. 6, pp. 500-504, 2006.

[3] M. L. Wang, "Exact solutions for a compound KdV-Burgers equation," Physics Letters A, vol. 213, no. 5-6, pp. 279-287, 1996.

[4] X. Li and M. Wang, "Applications of F-expansion to periodic wave solutions for KdV equation," Mathematica Applicata, vol. 18, no. 2, pp. 303-307, 2005.

[5] J. M. Burgers, "A mathematical model illustrating the theory of turbulence," in Advances in Applied Mechanics, vol. 1, Academic Press, New York, NY, USA, 1948.

[6] T. B. Benjamin, J. L. Bona, and J. J. Mahony, "Model equation for long water waves in nonlinear dispersive systems," Philosophical Transactions of the Royal Society of London Series A, vol. 272, pp. 47-78, 1972.

[7] V. E. Zakharov and E. A. Kuznetsov, "Three dimensional solitions ion acoustic solitary waves existence in nonisothermal plasma," Zhurnal Eksperimental'noi, vol. 66, no. 2, pp. 594-597, 1997.

where wave crest is $|(m-c+2 \omega) / 2 a m|$. 

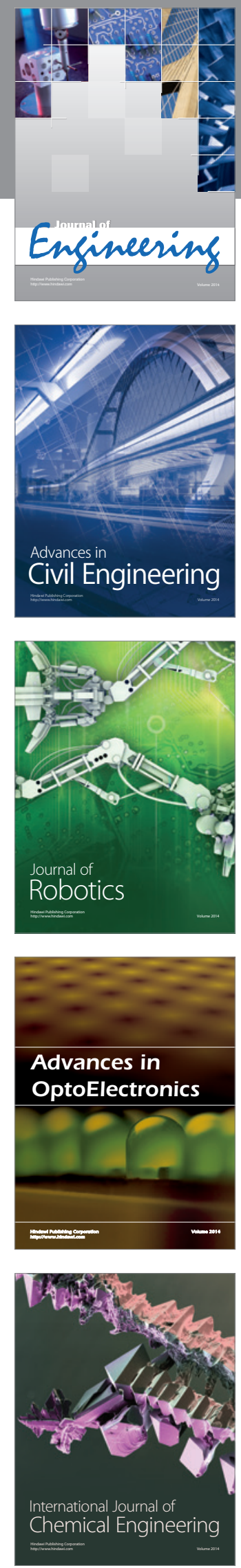

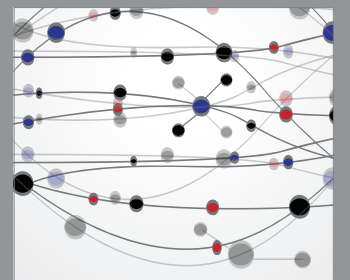

The Scientific World Journal
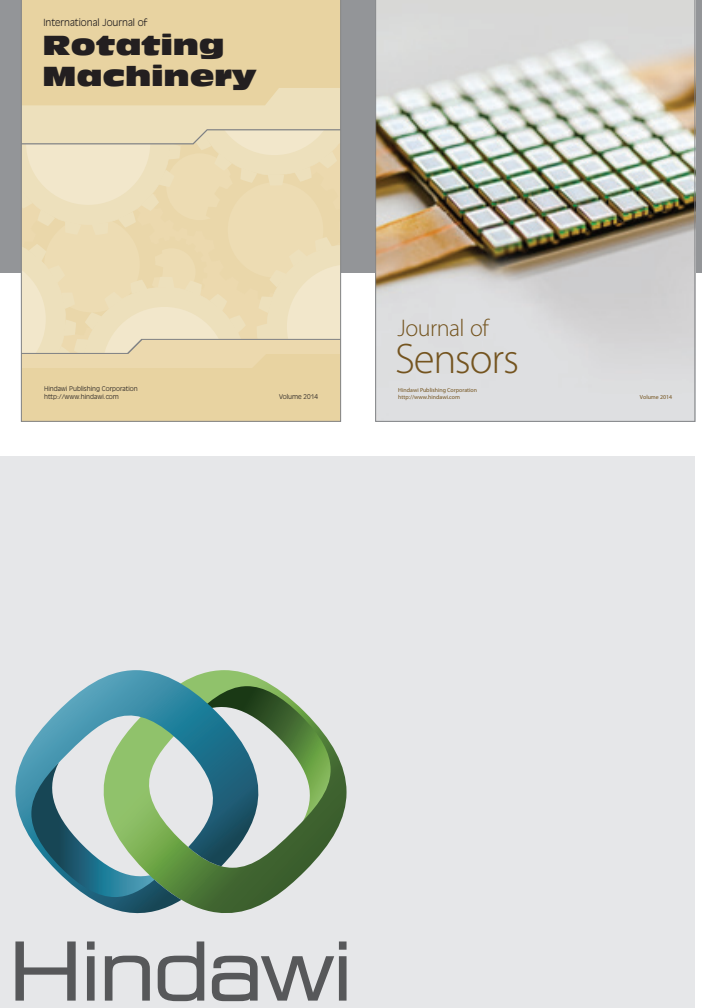

Submit your manuscripts at http://www.hindawi.com
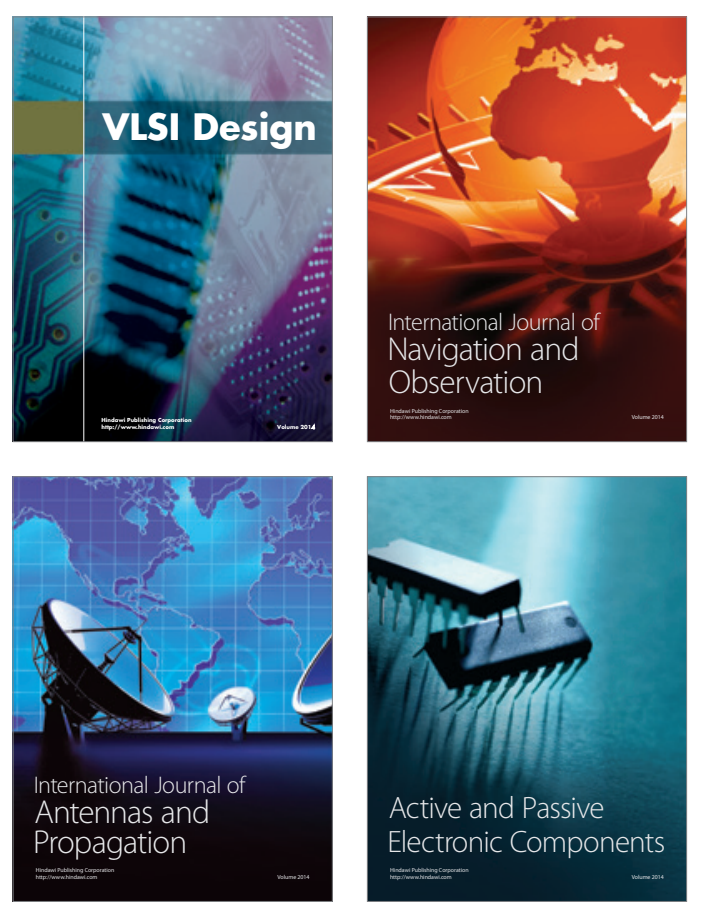
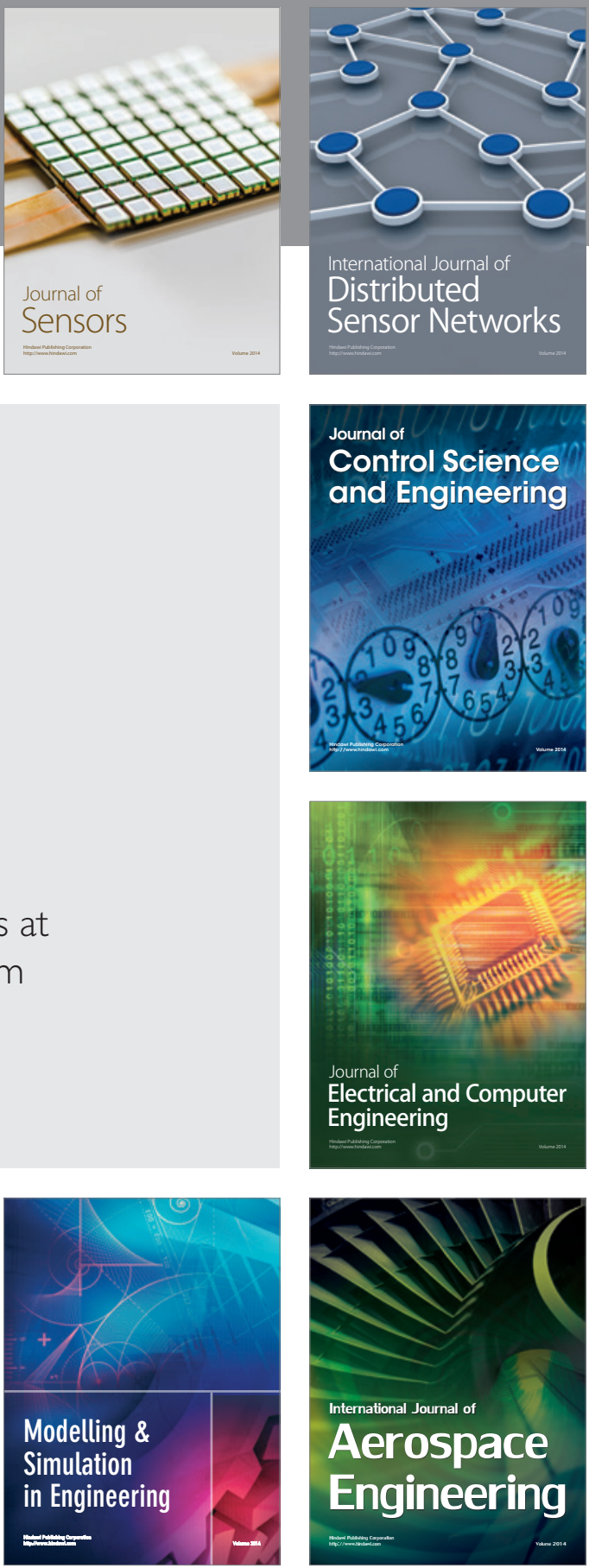

Journal of

Control Science

and Engineering
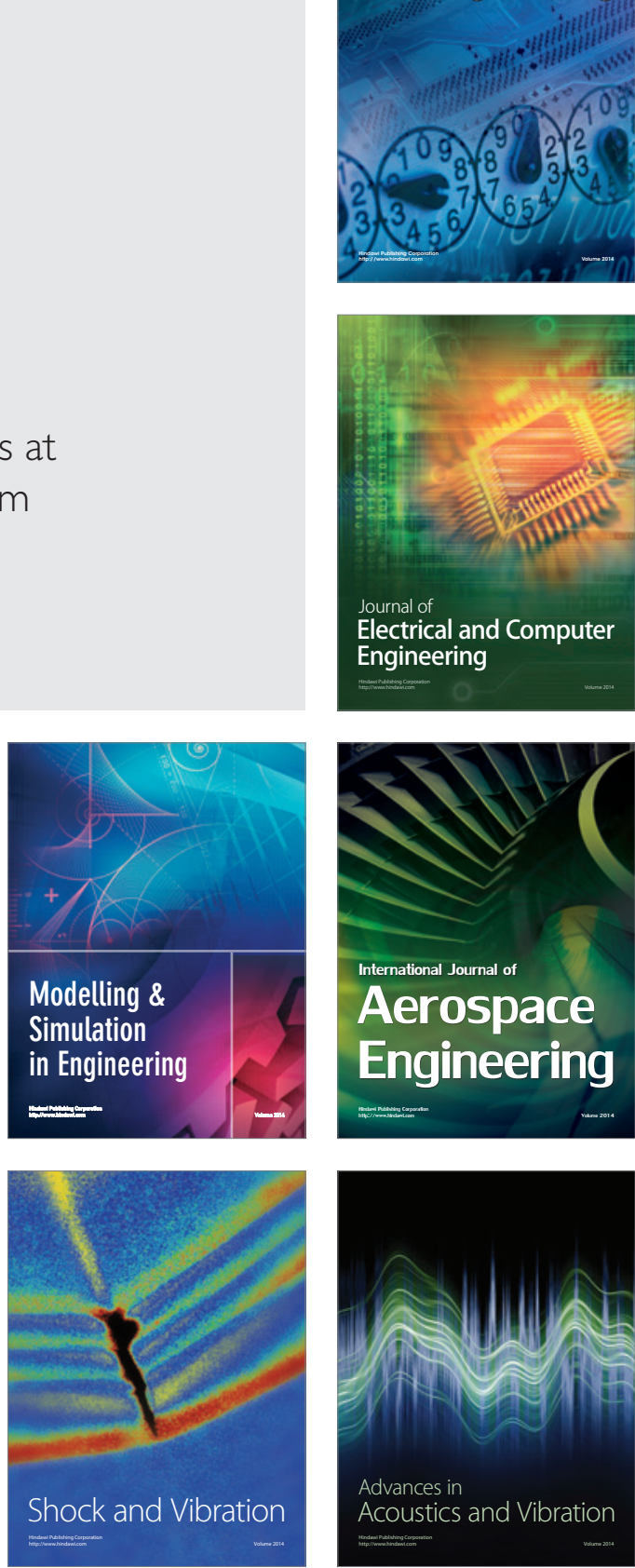